\title{
Erratum to: Synthesis and biologic activities of some novel heterocyclic chalcone derivatives
}

\author{
Punita Sharma - Suresh Kumar · Furqan Ali $\cdot$ Sumati Anthal $\cdot$ \\ Vivek K. Gupta - Inshad A. Khan · Surjeet Singh - Payare L. Sangwan • \\ Krishan A. Suri • Bishan D. Gupta · Devinder K. Gupta • Prabhu Dutt • \\ Ram A. Vishwakarma $\cdot$ Naresh K. Satti
}

Published online: 10 January 2013

(c) Springer Science+Business Media New York 2013

\section{Erratum to: Med Chem Res}

DOI 10.1007/s00044-012-0401-7

The original version of this article unfortunately contained one mistake. Here is the correction to it.

The name of a co-author, Furquan Ali is misspelled; the correct name is Furqan Ali.

The online version of the original article can be found under doi:10.1007/s00044-012-0401-7.

P. Sharma - P. L. Sangwan - K. A. Suri ·

B. D. Gupta · D. K. Gupta · P. Dutt

R. A. Vishwakarma · N. K. Satti $(\bowtie)$

Natural Product Chemistry Division, CSIR-Indian Institute

of Integrative Medicine, Canal Road, Jammu 180001, India

e-mail: nksatti@rediffmail.com

\section{S. Kumar}

Cancer Pharmacology Division, CSIR-Indian Institute

of Integrative Medicine, Canal Road, Jammu 18000, India

F. Ali · I. A. Khan

Clinical Microbiology Division, CSIR-Indian Institute

of Integrative Medicine, Canal Road, Jammu 180001, India

\section{S. Anthal · V. K. Gupta}

Department of Physics, University of Jammu,

Jammu 180006, India

S. Singh

Pharmacology Division, CSIR-Indian Institute of Integrative

Medicine, Canal Road, Jammu 18000, India 